\title{
Hamiltoniens quasi-convexes quasi-concaves
}

Revue française d'automatique, d'informatique et de recherche opérationnelle. Recherche opérationnelle, tome 25, no 4 (1991), p. 425-438.

$<$ http://www.numdam.org/item?id=RO_1991_25_4_425_0>

(C) AFCET, 1991, tous droits réservés.

L'accès aux archives de la revue « Revue française d'automatique, d'informatique et de recherche opérationnelle. Recherche opérationnelle » implique l'accord avec les conditions générales d'utilisation (http://www.numdam.org/ legal.php). Toute utilisation commerciale ou impression systématique est constitutive d'une infraction pénale. Toute copie ou impression de ce fichier doit contenir la présente mention de copyright.

\section{Numdam}

Article numérisé dans le cadre du programme

Numérisation de documents anciens mathématiques

http://www.numdam.org/ 


\title{
HAMILTONIENS QUASI-CONVEXES QUASI-CONCAVES (*)
}

\author{
par M. Atteia $\left({ }^{1}\right)$ et A. Elqortobi $\left({ }^{2}\right)$
}

\begin{abstract}
Résumé. - Notre objectif est de définir la polaire projective d'une fonction quasi-convexe quasiconcave. On utilisera dans notre étude la notion de polarité partielle d'une fonction quasi-convexe de plusieurs variables. On adopte donc le point de vue de Laurent. Cependant, comme on va le voir, ses résultats peuvent être étendus dans une autre direction.
\end{abstract}

Mots clés : Optimisation; fonction quasi-convexe quasi-concave; hamiltonien.

Abstract. - Our goal is to define the projective polar of a quasiconvex-quasiconcave function. In our study, we use the notion of partial polarity of a quasiconvex-quasiconcave function of multiple variables. Then we adopt the point of view of Laurent. However, his results can be extended in other direction.

Keywords : Optimization; quasiconvex quasiconcave function; hamiltonian.

\section{INTRODUCTION}

Dans cette partie, on va rappeler certaines définitions et propriétés qui nous seront utiles. Dans tout ce qui suit, $X$ et $X^{\prime}$ (resp. $Y$ et $Y^{\prime}$ ) sont deux espaces vectoriels topologiques localement convexes mis en dualité séparante par la forme bilinéaire qu'on notera de manière unique $\langle$,$\rangle .$

Définition 1: On dit qu'une fonction $f \in \overline{\mathbb{R}}^{X}$ est quasi-convexe (qcx) si : $\forall(u, v) \in X \times X, \forall \lambda \in[0,1], f[\lambda u+(1-\lambda) v] \leqq \operatorname{Max}[f(u), f(v)] . f$ est quasiconcave (qcv) si $-f$ est quasi-convexe.

$\left(^{*}\right)$ Reçu août 1990.

( ${ }^{1}$ ) Département de Mathématiques, Université Paul-Sabatier, Toulouse (France).

$\left({ }^{2}\right)$ Département de Mathématiques, Université Mohamed $1^{\text {er }}$, Faculté des Sciences, Oujda (Maroc) et Département de Mathématiques et d'Informatique, Université de Sherbrooke, Faculté des Sciences, Sherbrooke (Canada).

Recherche opérationnelle/Operations Research, 0399-0559/91/04 425 14/\$3.40

(c) AFCET-Gauthier-Villars 
On dit qu'une fonction $k \in \mathbb{R}^{X \times Y}$ est quasi-convexe quasi-concave (qcx-qcv) ou fonction selle si :

$\forall y \in Y$ la fonction $x \in X \mapsto k(x, y)$ est quasi-convexe et

$\forall x \in X$ la fonction $y \in Y \mapsto k(x, y)$ est quasi-concave.

Définition 2 : Soit $f \in \overline{\mathbb{R}}^{X}$.

Posons :

$$
\begin{gathered}
\forall x^{\prime} \in X^{\prime}, \quad f^{0}\left(x^{\prime}\right)=-\operatorname{Inf}\left\{f(x) /\left\langle x, x^{\prime}\right\rangle>1\right\}, \\
f^{\Delta}\left(x^{\prime}\right)=-\operatorname{Inf}\left\{f(x) /\left\langle x, x^{\prime}\right\rangle<1\right\},
\end{gathered}
$$

et

$$
\forall x \in X
$$

$f^{00}(x)=-\operatorname{Inf}\left\{f^{0}\left(x^{\prime}\right) /\left\langle x, x^{\prime}\right\rangle>1\right\}, \quad f^{\Delta \Delta}(x)=-\operatorname{Inf}\left\{f^{\Delta}\left(x^{\prime}\right) /\left\langle x, x^{\prime}\right\rangle<1\right\}$.

Les foctions $f^{\#}=\operatorname{Min}\left(f^{0}, f^{\Delta}\right)$ et $f^{\# \#}=\operatorname{Max}\left(f^{00}, f^{\Delta \Delta}\right)$ sont appelées respectivement polaire et bipolaire projectives de $f$.

Principaux résultats : ([1], [2])

(i) $f^{\# \# ~ e s t ~ l a ~ p l u s ~ g r a n d e ~ m i n o r a n t e ~ q u a s i-c o n v e x e ~ e t ~ s e m i-c o n t i n u e ~ i n f e ́ r i e u-~}$ rement (s.c.i.) de $f$; par suite, si $f$ est qcx et s.c.i., alors $f=f^{\sharp \#}$. En plus, $f \leqq g \Rightarrow f^{\# \#} \leqq g^{\# \#}$.

(ii) $f^{0}(0)=-\infty$ et $f^{\Delta}(0)=-\operatorname{Inf}\{f(x) / x \in X\}$.

En général $f^{\# \#} \neq\left(f^{\#}\right)^{\#}$, d'où on posera :

$$
f^{\# n}= \begin{cases}f^{\#} & \text { si } n \text { impair } \\ f^{\# \#} & \text { si } n \text { pair non nul. }\end{cases}
$$

Définition 3: Soit $f \in \overline{\mathbb{R}}^{X}$. On dira que $f$ vérifie la propriété $P(1)$ [resp. $P(2)]$ si $f(0)=-\infty$ (reps. $\forall x \in X, \forall \alpha \in[0,1] f(\alpha X) \geqq f(x))$.

LEMME 1 [10]: Soit $f$ une fonction qcx et s.c.i. appartenant $\grave{a} \overline{\mathbb{R}}^{X} . f$ vérifie $P(1) \Leftrightarrow f=f^{00}$ et $f$ vérifie $P(2) \Leftrightarrow f=f^{\Delta \Delta}$.

Remarque 1 : Comme $f^{0}=f^{000}$ (resp. $f^{\Delta}=f^{\Delta \Delta \Delta}$ ), on déduit que $f^{0}$ (resp. $f^{\Delta}$ ) vérifie $P(1)$ [resp. $P(2)$ ].

Définition 4 [10] : Soit une fonction qcx $f \in \overline{\mathbb{R}}^{X}$ et $x \in X . x^{\prime} \in X^{\prime}$ est un sousgradient de $f$ au point $x$ selon une polarité notée " $+»$ si et seulement si : $f^{+}\left(x^{\prime}\right)+f(x)=0$ et $x^{\prime}$ n'appartient pas à $\{x\}^{+}$. 
L'ensemble des sous-gradients de $f$ au point $x$ pour la polarité « + » s'appelle le sous-différentiel et sera noté $\partial f(x)$.

\section{POLARITÉ PARTIELLE D'UNE FONCTION}

Étant donnée une fonction $\mathrm{qcx}-\mathrm{qcv} k \in \overline{\mathbb{R}}^{X \times Y}$, nous aurons à faire certaines opérations de polarité par rapport à la première variable ou la seconde variable. On posera :

$$
\begin{array}{cc}
\forall x \in X & \text { (resp. } \left.\forall x^{\prime} \in X^{\prime}\right) \\
A(x)=\left\{x^{\prime} \in X^{\prime} /\left\langle x, x^{\prime}\right\rangle>1\right\} & \left(\text { resp. } A\left(x^{\prime}\right)=\left\{x \in X /\left\langle x, x^{\prime}\right\rangle>1\right\}\right)
\end{array}
$$

et

$$
B(x)=\left\{x^{\prime} \in X^{\prime} /\left\langle x, x^{\prime}\right\rangle<1\right\} \quad\left(\text { resp. } B\left(x^{\prime}\right)=\left\{x \in X /\left\langle x, x^{\prime}\right\rangle<1\right\}\right) .
$$

On notera aussi $A_{X} k$ et $B_{X} k$ [suivant qu'on utilise l'ensemble $A\left(x^{\prime}\right)$ ou $B\left(x^{\prime}\right)$ ], les fonctions de deux variables obtenues en appliquant l'opération de polarité par rapport à la première variable $x \in X$.

$$
\begin{aligned}
& A_{X} k\left(x^{\prime}, y\right)=-\operatorname{Inf}\left\{k(x, y) / x \in A\left(x^{\prime}\right)\right\} \\
& B_{X} k\left(x^{\prime}, y\right)=-\operatorname{Inf}\left\{k(x, y) / x \in B\left(x^{\prime}\right)\right\} .
\end{aligned}
$$

On sait que $A_{X} k$ et $B_{X} k$ sont qcx et s.c.i. par rapport à $x^{\prime}$ ([2]). Elles sont aussi qcx par rapport à $y$. En effet $A_{X} k$ et $B_{X} k$ sont les bornes supérieures d'une famille de fonctions qcx par rapport à $y$. En outre si $k$ est semicontinue supérieurement (s.c.s.) en $y$, alors $A_{X} k$ et $B_{X} k$ seront s.c.i. en $y$.

Pour simplifier notre étude, nous n'introduirons pas explicitement la notion de polaire d'une fonction qcv. Par contre l'opérateur d'inversion de signe $\theta$ nous permettra de transformer la fonction qcx-qcv $k$ en une fonction qcvqcx $\theta k(\theta k(x, y)=-k(x, y))$, et par suite d'appliquer l'opération de polarité à $\theta k$ par rapport à la seconde variable $y \in Y$.

$$
\begin{aligned}
& A_{Y} \theta k\left(x, y^{\prime}\right)=-\operatorname{Inf}\left\{-k(x, y) / y \in A\left(y^{\prime}\right)\right\}=\operatorname{Sup}\left\{k(x, y) / y \in A\left(y^{\prime}\right)\right\} \\
& B_{Y} \theta k\left(x, y^{\prime}\right)=-\operatorname{Inf}\left\{-k(x, y) / y \in B\left(y^{\prime}\right)\right\}=\operatorname{Sup}\left\{k(x, y) / y \in B\left(y^{\prime}\right)\right\}
\end{aligned}
$$

On constate aisément que $A_{Y} \theta k$ et $B_{Y} \theta k$ sont qcx et s.c.i. en $y^{\prime}$ et qcx en $x$. En outre, si $k$ est s.c.i. en $x$, alors $A_{Y} \theta k$ et $B_{Y} \theta k$ seront elles aussi s.c.i. 
en $x$. On posera aussi : $\forall(x, y) \in X \times Y$

$$
\begin{aligned}
& \sigma_{X} k(x, y)=\operatorname{Max}\left[A_{X^{\prime}} A_{X} k(x, y), B_{X^{\prime}} B_{X} k(x, y)\right] \\
& =\operatorname{Max}\left[\sup _{x^{\prime} \in A(x)} \inf _{u \in A\left(x^{\prime}\right)} k(u, y), \sup _{x^{\prime} \in B(x)} \inf _{u \in B\left(x^{\prime}\right)} k(u, y)\right] \\
& \begin{aligned}
\omega_{Y} k(x, y)=\operatorname{Min}\left[\theta A_{Y^{\prime}}, A_{Y} \theta\right. & \left.k(x, y), \theta B_{Y^{\prime}} B_{Y} \theta k(x, y)\right] \\
& =\operatorname{Min}\left[\inf _{y^{\prime} \in A(y)} \sup _{v \in A\left(y^{\prime}\right)} k(x, v) . \inf _{y^{\prime} \in B(y)} \sup _{v \in B\left(y^{\prime}\right)} k(x, v)\right.
\end{aligned}
\end{aligned}
$$

On obtient alors le résultat suivant :

Pour $y$ fixé (resp. pour $x$ fixé), $\sigma_{X} k(x, y)$ [resp. $\omega_{Y} k(x, y)$ ] est la plus grande minorante qcx et s.c.i. (resp. la plus petite majorante qcv et s.c.s.) de $k(x, y)$, ce qui veut dire : $\sigma_{X} k(x, y) \leqq k(x, y) \leqq \omega_{Y} k(x, y)$.

Dorénavant, pour alléger l'écriture, on remplacera les symboles $A_{X} k$ et $B_{X} k$ par l'unique symbole $P_{X} k$ et les ensembles $A(x)$ et $B(x)$ par l'unique ensemble $P(x)$. Ainsi, par exemple, les expressions analytiques (1) et (2) s'écriront de manière unique : $P_{X} k\left(x^{\prime}, y\right)=-\operatorname{Inf}\left\{k(x, y) / x \in P\left(x^{\prime}\right)\right\}$.

Les applications $\sigma$ et $\omega$ nous suggèrent d'introduire un opérateur de régularisation.

Défintion 5 : Soit une fonction qcx-qcv $k \in \overline{\mathbb{R}}^{X \times Y}$.

On appellera opération de régularisation par rapport à la variable $x \in X$, l'opération $\gamma_{X, P}$ définie par: $\gamma_{X, P} k=P_{X}, P_{X} k$. De même, on notera $\gamma_{Y, P}$ l'opération de régularisation par rapport à la variable $y: \gamma_{Y, P} k=\theta P_{Y}, P_{Y} \theta k$.

Exposons quelques méthodes pour construire des fonctions qcx-qcv. Soit $\varphi$ une fonction qcx et s.c.i. de $\overline{\mathbb{R}}^{X^{\prime} \times Y}$. Notons par $\psi_{P}$ la fonction qcx et s.c.i. de $\overline{\mathbb{R}}^{X^{\prime} \times Y^{\prime}}$ définie par :

$$
\psi_{P}\left(x^{\prime}, y\right)=-\operatorname{Inf}\left\{\varphi\left(x, y^{\prime}\right) /\left(x, y^{\prime}\right) \in P\left(x^{\prime}\right) \times P(y)\right\} .
$$

En utilisant les notations précédentes, on obtient :

$$
\psi_{P}=P_{X} \theta P_{Y}, \varphi=P_{Y}, \theta P_{X} \varphi
$$

La polaire projective de $\varphi$ est alors : $\psi=\operatorname{Min}\left(\psi_{A}, \psi_{B}\right)$. Comme $\varphi$ est qcx et s.c.i., on a : $\varphi^{\sharp \#}=\varphi$. Posons :

$$
\varphi_{P}\left(x, y^{\prime}\right)=-\operatorname{Inf}\left\{\psi_{P}\left(x^{\prime}, y\right) /\left(x^{\prime}, y\right) \in P(x) \times P\left(y^{\prime}\right)\right\} .
$$

On obtient alors : $\varphi_{P}=P_{X^{\prime}}, \theta P_{Y} \psi_{P}=P_{Y} \theta P_{X^{\prime}} \psi_{P}$ et $\varphi=\operatorname{Max}\left(\varphi_{A}, \varphi_{B}\right)$. 
THÉORÈME $1:$ Les fonctions $\vec{k}_{P}=\theta P_{Y^{\prime}} \varphi_{P}$ et $\underline{k}_{P}=P_{X^{\prime}}, \psi_{P}$ sont $q c x-q c v$ sur $X \times Y$.

Preuve: Soient $(x, y) \in X \times Y \cdot \bar{k}_{P}(x, y)=\operatorname{Inf}\left\{\varphi_{P}\left(x, y^{\prime}\right) / y^{\prime} \in P(y)\right\}$ donc $\bar{k}_{P}(x,$.$) est qcv sur Y$. Montrons que $\bar{k}_{P}(., y)$ est qvx sur $X$. Soient $(u, v) \in X^{2}$ et $\lambda \in] 0,1\left[\right.$. Si $\bar{k}_{P}(u, y)=+\infty$ où $\vec{k}_{P}(v, y)=+\infty$ alors d'une manière évidente $\bar{k}_{P}[\lambda u+(1-\lambda) v, y] \leqq \operatorname{Max}\left[\bar{k}_{P}(u, y), \bar{k}_{P}(v, y)\right]$.

Supposons que $\bar{k}_{P}(u, y)<+\infty$ et $\bar{k}_{P}(v, y)<+\infty$.

Considérons deux nombres réels $a$ et $b$ vérifiant : $\bar{k}_{P}(u, y)<a$ et $\bar{k}_{P}(v, y)<b$. $\exists\left(y^{\prime}, z^{\prime}\right) \in[P(y)]^{2}$ tels que : $\bar{k}_{P}(u, y) \leqq \varphi_{P}\left(u, y^{\prime}\right) \leqq a$ et $\bar{k}_{P}(v, y) \leqq \varphi_{P}\left(v, z^{\prime}\right) \leqq b$. Comme $P(y)$ est un ensemble convexe, $\lambda y^{\prime}+(1-\lambda) z^{\prime} \in P(y)$. Sachant que $\varphi_{P}$ est qcx,

$$
\begin{aligned}
& k_{P}[\lambda u+(1-\lambda) v, y] \leqq \varphi_{P}[\lambda u+(1-\lambda) v\left.\lambda y^{\prime}+(1-\lambda) z^{\prime}\right] \\
& \leqq \\
& \operatorname{Max}\left[\varphi_{P}\left(u, y^{\prime}\right), \varphi_{P}\left(v, z^{\prime}\right)\right] \leqq \operatorname{Max}(a, b) .
\end{aligned}
$$

En faisant tendre $(a, b)$ vers $\left[\bar{k}_{P}(u, y), \bar{k}_{P}(v, y)\right]$, on déduit que $\bar{k}_{P}(., y)$ est qvx. Quant à l'autre résultat, il suffit de remarquer que $\psi_{P}$ est qcx sur $X \times Y$ et on emploie le même raisonnement que précédemment. @

Lemme $2: P_{Y} P_{Y}, \varphi_{P}=\varphi_{P}$ et $P_{X} P_{X^{\prime}}, \psi_{P}=\psi_{P}$.

Preuve: Par construction, $\varphi_{A}$ (resp. $\varphi_{B}$ ) est qcx et s.c.i. D'après la remarque 1, elle vérifie la propriété (P1) [resp. (P2)]. Même chose pour $\psi$. @

Notation: $S_{P}(X \times Y)$ désignera l'ensemble des fonctions $f \in \overline{\mathbb{R}}^{X \times Y}$ qui sont qcx par rapport aux deux variables et qui vérifient les propriétés suivantes: $f=P_{X}, P_{X} f$ et $f=P_{Y}, P_{Y} f$.

THÉORÈME 2 : Soient $\varphi \in \overline{\mathbb{R}}^{X \times Y} q c x$ et s.c.i., $\varphi_{P}$ et $\psi_{P}$ construits comme précédemment. Alors pour toute fonction qcx-qcv $k$ vérifiant $\underline{k}_{P} \leqq k \leqq k_{P}$, on $a$ : $\gamma_{X, P} k=\underline{k}_{P}, \gamma_{Y, P} k=\bar{k}_{P}, P_{X} k=\psi_{P}$ et $P_{Y} \theta k=\varphi_{P}$.

Preuve :

$$
\gamma_{X, P} \bar{k}_{P}=P_{X}, P_{X} \bar{k}_{P}=P_{X}, P_{X} \theta P_{Y}, \varphi_{P}=P_{X}, \psi_{P}=\underline{k}_{P}
$$

et

$$
\gamma_{Y, P} \underline{k}_{P}=\theta P_{Y}, P_{Y} \theta \underline{k}_{P}=\theta P_{Y}, P_{Y} \theta P_{X}, \psi=\theta P_{Y}, \varphi_{P}=\bar{k}_{P} .
$$

Par suite $\forall k$ tel que

$$
\begin{gathered}
\underline{k}_{P} \leqq k \leqq \bar{k}_{P}, \quad \gamma_{X, P} k=\underline{k}_{P} \quad \text { et } \quad \gamma_{Y, P} k=\bar{k}_{P} . \\
P_{X} \underline{k}_{P}=P_{X} P_{X^{\prime}} \psi_{P}=\psi_{P}
\end{gathered}
$$

vol. $25, n^{\circ} 4,1991$ 
et

$$
\begin{gathered}
P_{X} \bar{k}_{P}=P_{X} \theta P_{Y}, \varphi_{P}=\psi_{P} \Rightarrow P_{X} k=\psi_{P} \\
P_{Y} \theta \bar{k}_{P}=P_{Y} P_{Y^{\prime}} \varphi_{P}=\varphi_{P}
\end{gathered}
$$

et

$$
P_{Y} \theta \underline{k}_{P}=P_{Y} \theta P_{X}, \psi_{P}=\varphi_{P} \quad \Rightarrow \quad P_{Y} \theta k=\varphi_{P}
$$

\section{DÉfinition 6 : Soit $k \in \overline{\mathbb{R}}^{X \times Y}$.}

On dira que $k$ est de type $S_{P}$ si $k$ est qcx-qcv et vérifie les deux conditions suivantes : $\gamma_{X, P} \gamma_{Y, P} k=\gamma_{X, P} k$ et $\gamma_{Y, P} \gamma_{X, P} k=\gamma_{Y, P} k$.

Théorème $3:$ Soit $k \in \overline{\mathbb{R}}^{X \times Y}$ de type $S_{P}$. Alors il existe deux fonctions quasiconvexes $\varphi_{P}$ et $\psi_{P}$ vérifiant: $P_{Y} \theta P_{X}, \psi_{P}=\varphi_{P}$ et $P_{X} \theta P_{Y}, \varphi_{P}=\psi_{P}$, telles que si l'on pose $\underline{k}_{P}=P_{X}, \psi_{P}$ et $\bar{k}_{P}=\theta P_{Y^{\prime}}, \varphi_{P}$, on ait $\underline{k}_{P} \leqq k \leqq \bar{k}_{P}$. De plus on $a$ : $\underline{k}_{P}=\gamma_{X, P} k$ et $\bar{k}_{P}=\gamma_{Y, P} k$. Enfin, $\varphi_{P} \in S_{P}\left(X \times Y^{\prime}\right)$ et $\psi_{P} \in S_{P}\left(X^{\prime} \times Y\right)$.

Preuve : On posera $k_{x}=\gamma_{X, P} k$ et $k_{y}=\gamma_{Y, P} k$ s'il n'y a pas d'ambigüités. On a évidemment $k_{x} \leqq k \leqq k_{y}$. Comme $k$ est de type $S_{P}$, on a :

$$
\gamma_{Y, P} k_{x}=\gamma_{Y, P} \gamma_{X, P} k=\gamma_{Y, P} k=k_{y} \quad \text { et } \quad \gamma_{X, P} k_{y}=\gamma_{X, P} \gamma_{Y, P} k=\gamma_{X, P} k=k_{x}
$$

donc $\gamma_{Y, P} k_{x}=k_{y}$ et $\gamma_{X, P} k_{y}=k_{x}$. Par suite, $\forall k^{\prime}$ tel que $k_{x} \leqq k^{\prime} \leqq k_{y}$, on a :

$$
\gamma_{Y, P} k^{\prime}=k_{y} \quad \text { et } \quad \gamma_{X, P} k^{\prime}=k_{x} \text {. }
$$

Posons : $P_{Y} \theta k_{x}=\varphi_{P}$ et $P_{X} k_{y}=\psi_{P}$. On obtient :

$$
P_{X} \theta P_{Y}, \varphi_{P}=P_{X} \theta P_{Y}, P_{Y} \theta k_{x}=P_{X} \gamma_{Y, P} k_{x}=P_{X} k_{y}=\psi_{P}
$$

et

$$
P_{Y} \theta P_{X}, \psi_{P}=P_{Y} \theta P_{X}, P_{X} k_{y}=P_{Y} \theta \gamma_{X, P} k_{y}=P_{Y} \theta k_{x}=\varphi_{P}
$$

Calculons $\underline{k}_{P}$ et $\bar{k}_{P}$ :

$\underline{k}_{P}=P_{X}, \psi_{P}=P_{X}, P_{X} k_{y}=\gamma_{X, P} k_{y}=k_{x}, k_{P}=\theta P_{Y}, \varphi_{P}=\theta P_{Y^{\prime}} P_{Y} \theta k_{x}=\gamma_{Y, P} k_{x}=k_{y}$.

Par construction $\varphi$ et $\psi$ sont deux fonctions qcx par rapport aux deux variables. Pour montrer que $\varphi_{P} \in S_{P}\left(X \times Y^{\prime}\right)$ et $\psi_{P} \in S_{P}\left(X^{\prime} \times Y\right)$, il suffit de remarquer que pour toute fonction $\operatorname{qcx} f \in \overline{\mathbb{R}}^{X}$ on a : $P_{X} P_{X^{\prime}} P_{X} f=P_{X} f$ (cf. [1], [2] où la remarque 1). Par conséquent,

$$
P_{X} P_{X}, \psi_{P}=P_{X} P_{X}, P_{X} k_{y}=P_{X} k_{y}=\psi_{P}
$$

et

$$
P_{Y} P_{Y}, \varphi_{P}=P_{Y} P_{Y}, P_{Y} \theta k_{x}=P_{Y} \theta k_{x}=\varphi_{P}
$$


Comme $\varphi_{P}$ et $\psi_{P}$ peuvent s'écrire aussi de la manière suivante :

$$
\varphi_{P}=P_{X}, \theta P_{Y} \psi_{P} \quad \text { et } \quad \psi_{P}=P_{Y}, \theta P_{X} \varphi_{P},
$$

on a :

$$
P_{X^{\prime}} P_{X} \varphi_{P}=P_{X^{\prime}}, P_{X} P_{X^{\prime}}, \theta P_{Y} \psi_{P}=P_{X^{\prime}}, \theta P_{Y} \psi_{P}=\varphi_{P}
$$

et

$$
P_{Y}, P_{Y} \psi_{P}=P_{Y}, P_{Y} P_{Y}, \theta P_{X}, \varphi_{P}=P_{Y}, \theta P_{X} \varphi_{P}=\psi_{P} .
$$

Les théorèmes (2) et (3) suggèrent de construire une classe d'équivalence dont les éléments extrêmes seront $\underline{k}_{P}$ et $\bar{k}_{P}$.

DéfINITION 7 : Soient deux fonctions qcx-qcv $k$ et $k^{\prime} \in \overline{\mathbb{R}}^{X \times Y}$ et $\mathbb{R}_{P}$ une relation définie par: $k \mathbb{R}_{P} k^{\prime} \Leftrightarrow \gamma_{X, P} k=\gamma_{X, P} k^{\prime}$ et $\gamma_{Y, P} k=\gamma_{Y, P} k^{\prime}$.

THÉORÈME $4: \mathbb{R}_{P}$ est une classe d'équivalence. Si $\underline{k}_{P}$ et $\bar{k}_{P}$ sont les fonctions obtenues précédemment, alors

$$
K_{P}=\left\{k \in \overline{\mathbb{R}}^{X \times Y} q c x \text {-qcv telles que } \underline{k}_{P} \leqq k \leqq \bar{k}_{P}\right\}
$$

est une classe d'équivalence.

Preuve : Il est clair que $\mathbb{R}_{P}$ est une classe d'équivalence. En plus tous les éléments de $K_{P}$ sont équivalents entre eux. En effet, soient $k$ et $k^{\prime} \in K_{P}$. D'après le théorème (2), $\gamma_{X, P} k=\gamma_{X, P} k^{\prime}=\underline{k}_{P}$ et $\gamma_{Y, P} k=\gamma_{Y, P} k^{\prime}=\bar{k}_{P}$. Supposons que $k^{\prime}$ soit équivalent à $k \in K_{P}$. On a donc $\gamma_{X, P} k=\gamma_{X, P} k^{\prime}=\underline{k}_{P}$ d'où $\underline{k}_{P} \leqq k^{\prime}$. Enfin $\gamma_{Y, P} k=\gamma_{Y, P} k^{\prime}=\bar{k}_{P}$ d'où $k^{\prime} \leqq \bar{k}_{P}$. On a donc bien $k^{\prime} \in K_{P}$.

Remarque 2 : Toutes les fonctions qcx-qcv $k$ situées entre $\underline{k}_{P}$ et $\vec{k}_{P}$ sont de type $S_{P}$. Dans toute la suite, nous ne considérerons que des fonctions qui sont de type $S_{P}$. Si $k$ est de type $S_{P}$, la classe de $k$ est donc l'ensemble $K_{P}$ des fonctions qcx-qcv $k^{\prime}$ comprises entre $\underline{k}_{P}=\gamma_{X, P} k$ et $\vec{k}_{P}=\gamma_{Y, P} k$.

\section{POLAIRES D'UNE FONCTION QUASI-CONVEXE QUASI-CONCAVE}

Soit $k$ de type $S_{P}$. On notera $K_{P}$ la classe de $k$.

(i) Appliquons l'opération de polarité par rapport à la première variable $x \in X$. On obtient une fonction $P_{X} k \in \mathbb{\mathbb { R }}^{X^{\prime} \times Y}$ qui est qcx par rapport aux deux variables. Appliquons ensuite l'opération de polarité par rapport à la seconde variable $y \in Y$ et inversons le signe pour obtenir à nouveau une fonction qcxqcv définie sur $X^{\prime} \times Y^{\prime}$ que l'on notera $k_{P}^{*}$ et sera appelée polaire supérieure 
de $k$ par rapport à $P$. On obtient : $k_{P}^{*}=\theta P_{Y} P_{X} k$ c'est-à-dire

$k_{P}^{*}\left(x^{\prime}, y^{\prime}\right)=\operatorname{Inf}\left\{-\operatorname{Inf}\left\{k(x, y) / x \in P\left(x^{\prime}\right)\right\} / y \in P\left(y^{\prime}\right)\right\}=-\operatorname{Sup}_{y \in P\left(y^{\prime}\right)} \operatorname{Inf}_{x \in P\left(x^{\prime}\right)} k(x, y)$.

(ii) Appliquons maintenant l'opération de polarité par rapport à la seconde variable $y \in Y$ (après avoir inversé le signe). On obtient une fonction $P_{Y} \theta k \in \overline{\mathbb{R}}^{X \times Y^{\prime}}$ qui est qcx par rapport aux deux variables. Appliquons ensuite l'opération de polarité par rapport à la première variable $x \in X$. On obtient encore une fonction qcx-qcv définie sur $X^{\prime} \times Y^{\prime}$ que l'on notera $k_{P^{*}}$ et sera appelée polaire inférieure de $k$ par rapport $\grave{a} P$. On obtient : $k_{P^{*}}=P_{X} P_{Y} \theta k$ c'est-à-dire

$$
\begin{aligned}
k_{P^{*}}\left(x^{\prime}, y^{\prime}\right)=-\operatorname{Inf}\left\{-\operatorname{Inf}\left\{-k(x, y) / y \in P\left(y^{\prime}\right)\right\} / x \in P\left(x^{\prime}\right)\right\} & \\
& =-\operatorname{Inf}_{x \in P\left(x^{\prime}\right)} \operatorname{Sup}_{y \in P\left(y^{\prime}\right)} k(x, y)
\end{aligned}
$$

On a ainsi obtenu deux fonctions qcx-qcv $k_{P}^{*}$ et $k_{P^{*}}$ de type $S_{\mathrm{P}}$ dont la seule différence dans leur expression est l'interversion des opérations Inf et Sup. On a trivialement : $\forall\left(x^{\prime}, y^{\prime}\right) \in X^{\prime} \times Y^{\prime} k_{P^{*}}\left(x^{\prime}, y^{\prime}\right) \leqq k_{P}^{*}\left(x^{\prime}, y^{\prime}\right)$.

DéfInITION 8 : L'application $k^{*}=\operatorname{Min}\left(k_{A}^{*}, k_{B}^{*}\right)$ [resp. $k_{*}=\operatorname{Min}\left(k_{A^{*}}, k_{B^{*}}\right)$ ] est appelée polaire projective supérieure (resp. inférieure) de $k$.

Remarque 3 : On a toujours l'inégalité $k_{*} \leqq k^{*}$.

$\mathrm{Au}$ théorème (2), à partir de deux fonctions, on a obtenu $\underline{k}_{P}$ et $\bar{k}_{P}$ par polarité partielle. On peut recommencer cette construction mais en inversant les rôles de $\psi_{P}$ et $\varphi_{P}$. A partir de $\varphi_{P} \in S_{P}\left(X \times Y^{\prime}\right)$ et $\psi_{P} \in S_{P}\left(X^{\prime} \times Y\right)$ telles que $\varphi_{P}=P_{X}, \theta P_{Y} \psi_{P}$ et $\psi_{P}=P_{Y}, \theta P_{X} \varphi_{P}$, on posera $\bar{m}_{P}=\theta P_{Y} \psi_{P}$ et $\underline{m}_{P}=P_{X} \varphi_{P}$. On constate alors que $\bar{m}_{P}=k_{P}^{*}$ et $\underline{m}_{P}=k_{P *}$. En effet $\bar{m}_{P}=\theta P_{Y} P_{X} k=k_{P}^{*}$ car $\psi_{P}=P_{X} k$ et $m_{P}=P_{X} P_{Y} \theta k=k_{P *}$ car $\varphi_{P}=P_{Y} \theta k$ ( $c f$. théorème 2). Ainsi les éléments $k_{P}^{*}$ et $k_{P^{*}}$ sont les éléments extrêmes d'une classe. On notera $M_{P}$ l'ensemble des fonctions qcx-qcv $m$ définies sur $X^{\prime} \times Y^{\prime}$ telles que $\underline{m}_{P} \leqq m \leqq \bar{m}_{P}$. Comme les fonctions $\varphi_{P}$ et $\psi_{P}$ sont les mêmes quelle que soit la fonction qcx-qcv que l'on prend dans la classe $K_{P}$, il en est de même pour $\underline{m}$ et $\bar{m}$. Ainsi $M_{P}$ dépend en fait de $K_{P}$. On dira que $M_{P}$ est la classe polaire de $K_{P}$ et l'on notera $M_{P}=K_{P}^{*}$.

Pour $m \in M_{P}$ ( $m$ est donc de type $S_{P}$ ), on peut définir de la même façon $m_{P}^{*}=\theta P_{Y}, P_{X}, m$ et $m_{P^{*}}=P_{X}, P_{Y}, \theta m$, et l'on notera $M_{P}^{*}=K_{P}^{* *}$ l'ensemble des fonctions qcX-qcv comprises entre $m_{P^{*}}$ et $m_{P}^{*}$, d'où le résultat suivant.

ThÉORÈme 5 : Soient $k \in \widehat{\mathbb{R}}^{X \times Y}$ de type $S_{P}$ et $K_{P}$ la classe de $k$. 
On a alors $K_{P}^{* *}=K_{P}$. Plus précisément si $\underline{k}_{P}=\gamma_{X, P} k$ et $\bar{k}_{P}=\gamma_{Y, P} k$ alors $\forall m \in K_{P}^{*}$ (c'est-à-dire vérifiant $k_{P^{*}} \leqq m \leqq k_{P}^{*}$ ), on a $: m_{P}^{*}=\bar{k}_{P}$ et $m_{P^{*}}=\underline{k}_{P}$.

Preuve : Il suffit de prouver que

$$
\left(k_{P^{*}}\right)^{*}=k_{P}^{* *}=\bar{k}_{P} \quad \text { et } \quad\left(k_{P}^{*}\right)_{*}=k_{P}^{* *}=\underline{k}_{P} .
$$

Soient $\psi_{P}=P_{X} \gamma_{Y, P} k$ et $\varphi_{P}=P_{Y} \theta \gamma_{X, P} k$. On a alors $\psi_{P}=P_{X} \bar{k}_{P}$ et $\varphi_{P}=P_{Y} \theta \underline{k}_{P}$. Comme

$$
\underline{k}=\gamma_{X, P} k=\gamma_{X, P} \gamma_{Y, P} k=P_{X}, P_{X} \gamma_{Y, P} k=P_{X}, \psi_{P}
$$

et

$$
k_{P}=\gamma_{Y, P} k=\gamma_{Y, P} \gamma_{X, P} k=\theta P_{Y}, P_{Y} \theta \gamma_{X, P} k=\theta P_{Y}, \varphi_{P},
$$

il s'ensuit que $\psi_{P}=P_{X} k$ et $\varphi_{P}=P_{Y} \theta k$ (cf. théorème 2), d'où $k_{P^{*}}=P_{X} \varphi_{P}$ et $k_{P}^{*}=\theta P_{Y} \psi_{P}$.

(i) En posant $\underline{m}_{P}=k_{P^{*}}$, on a :

$$
\underline{m}_{P}^{*}=\theta P_{Y}, P_{X^{\prime}} \underline{m}_{P}=\theta P_{Y^{\prime}} P_{X^{\prime}} P_{X} \varphi_{P}=\theta P_{Y^{\prime}} \varphi_{P}=k_{P} .
$$

Si on pose $\bar{m}_{P}=k_{P}^{*}$, alors :

$$
\bar{m}_{P}^{*}=\theta P_{Y}, P_{X}, \bar{m}_{P}=\theta P_{X^{\prime}}, P_{X}, \theta P_{Y} \psi_{P}=\theta P_{Y}, \varphi_{P}=\bar{k}_{P} .
$$

Par conséquent $\forall m$ tel que $\underline{m}_{P} \leqq m \leqq \bar{m}_{P}: m_{P}^{*}=\bar{k}_{P}$.

(ii) $\bar{m}_{P *}=P_{X} P_{Y}, \theta \bar{m}_{P}=P_{X}, P_{Y}, \theta \theta P_{Y} \psi_{P}=P_{X}, \psi_{P}=\underline{k}_{P}$. Enfin

$$
\begin{aligned}
\underline{m}_{P^{*}}=P_{X^{\prime}}, P_{Y^{\prime}}, \theta \underline{m}_{P} \Rightarrow \quad \underline{m}_{P^{*}}=P_{X^{\prime}} P_{Y^{\prime}} \theta P_{X} \varphi_{P} \\
\quad=P_{X^{\prime}} \psi_{P}=\underline{k}_{P} \Rightarrow \forall m: \underline{m}_{P} \leqq m \leqq \bar{m}_{P}, m_{P^{*}}=\underline{k}_{P} .
\end{aligned}
$$

\section{Application. Problème quasi-convexe quasi-concave}

Soient $h \in \overline{\mathbb{R}}^{X \times Y}$ une fonction qcx-qcv et $\left(u^{\prime}, v^{\prime}\right) \in X^{\prime} \times Y^{\prime}$. Posons :

$$
f(x)=\operatorname{Max}\left[\operatorname{Sup}_{y \in A\left(v^{\prime}\right)} h(x, y), \operatorname{Sup}_{y \in B\left(v^{\prime}\right)} h(x, y)\right]
$$

et

$$
g(y)=\operatorname{Min}\left[\operatorname{Inf}_{x \in A\left(u^{\prime}\right)} h(x, y), \operatorname{Inf}_{x \in B\left(u^{\prime}\right)} h(x, y)\right]
$$

Prenons

$$
\left(u^{\prime}, v^{\prime}\right)=(0,0): f(x)=\operatorname{Max}\left[-\infty, \operatorname{Sup}_{y \in Y} h(x, y)\right]=\operatorname{Sup}_{y \in Y} h(x, y)
$$


et

$$
g(y)=\operatorname{Min}\left[+\infty, \operatorname{Inf}_{x \in X} h(x, y)\right]=\underset{x \in X}{\operatorname{Inf}} h(x, y)
$$

Définition 9: Soit $h \in \overline{\mathbb{R}}^{X \times Y}$. Au triplet $[h, X, Y]$ on associe les deux problèmes :

$$
\begin{aligned}
& a=\operatorname{Inf}_{x \in X} f(x) \quad \text { avec } \\
& f(x)=\operatorname{Sup}_{y \in Y} h(x, y) \\
& b=\operatorname{Sup}_{y \in Y} g(x) \quad \text { avec } \quad g(y)=\operatorname{Inf}_{x \in X} h(x, y) .
\end{aligned}
$$

On conviendra d'appeler $(\mathrm{P})$ le problème primal et $(\mathrm{Q})$ le problème dual.

A partir de maintenant, $P_{X}$ représentera uniquement l'opération $B_{X}$ et $P(x)$ l'ensemble $B(x)$. De même, on écrira $(k, S, \varphi, \psi, \ldots)$ au lieu de $\left(k_{P}, S_{P}, \varphi_{P}, \psi_{P}, \ldots\right)$.

THÉORĖME 6 : Soient les fonctions $\varphi, \psi, \underline{k}$ et $\bar{k}$ vérifiant les hypothèses $d u$ théorème (2). Alors toutes les fonctions qcx-qcv $k$ vérifiant $\underline{k} \leqq k \leqq k$, ont les mêmes problèmes primal et dual associés.

Preuve : Soit $k$ arbitraire vérifiant $\underline{k} \leqq k \leqq k$. On a :

$$
f(x)=\operatorname{Sup}\{k(x, y) / y \in Y\}=P_{Y} \theta k\left(x, 0_{Y^{\prime}}\right) .
$$

En effet :

$$
P_{Y} \theta k\left(x, 0_{Y^{\prime}}\right)=-\operatorname{Inf}\left\{-k(x, y) / y \in P\left(0_{Y^{\prime}}\right)\right\} .
$$

Comme $P\left(0_{Y^{\prime}}\right)=Y$, il s'ensuit que $f(x)=P_{Y} \theta k\left(x, 0_{Y^{\prime}}\right)=\varphi\left(x, 0_{Y^{\prime}}\right)$. De même $\theta P_{X} k\left(0_{X^{\prime}}, y\right)=\operatorname{Inf}\left\{k(x, y) / x \in P\left(0_{X}\right)\right\}$. Comme $P\left(0_{X^{\prime}}\right)=X$, il s'ensuit que $g(x)=\theta P_{X} k\left(0_{X^{\prime}}, y\right)=-\psi\left(0_{X^{\prime}}, y\right)$.

On constate que pour tout $k$ vérifiant $k \leqq k \leqq k$, on a les mêmes fonctions $f$ en $g$, donc les mêmes problèmes primal et dual. @

Définition 10 : On dit que le triplet $[h, X, Y]$ a une valeur $v$ si l'on a l'égalité suivante :

$$
v=\operatorname{Sup}_{y \in Y} \operatorname{Inf}_{x \in X} h(x, y)=\operatorname{Inf}_{x \in X} \operatorname{Sup}_{y \in Y} h(x, y) .
$$

ThÉorème 7: Soient $k \in \mathbb{\mathbb { R }}^{X \times Y}$ de type $S$ et $K$ la classe de $k$. Le triplet $[k, X, Y]$ a une valeur v si et seulement si $k_{*}\left(0_{X^{\prime}}, 0_{Y^{\prime}}\right)=k^{*}\left(0_{X^{\prime}}, 0_{Y^{\prime}}\right) c^{\prime}$ 'est-à-dire que toutes les fonctions $m \in K^{*}$ prennent la même valeur en $\left(0_{X^{\prime}}, 0_{Y^{\prime}}\right) \in X^{\prime} \times Y^{\prime}$. 
Preuve : On a : $k^{*}=\theta P_{Y} P_{X} k$ et $k_{*}=P_{X} P_{Y} \theta k$, c'est-à-dire :

$$
\begin{array}{r}
k_{*}\left(0_{X^{\prime}}, 0_{Y^{\prime}}\right)=-\operatorname{Inf}\left\{\operatorname{Sup}\{k(x, y) / y \in Y\} / x \in P\left(0_{X^{\prime}}\right)\right\} \\
\quad=-\operatorname{Inf}_{x \in X} \operatorname{Sup}_{y \in Y} k(x, y)=-a \\
\begin{array}{r}
k^{*}\left(0_{X^{\prime}}, 0_{Y^{\prime}}\right)=\operatorname{Inf}\left\{-\operatorname{Inf}\{k(x, y) / x \in X\} / y \in P\left(0_{Y^{\prime}}\right)\right\} \\
=-\operatorname{Sup}_{y \in Y} \operatorname{Inf}_{x \in X} k(x, y)=-b . \quad @ .
\end{array}
\end{array}
$$

Remarque 4: On ne peut trouver de résultats pareils si on remplace $P_{X}$ par $A_{X}$ et l'ensemble $P(x)$ par $A(x)$ car $A\left(0_{X^{\prime}}\right)=A\left(0_{Y}\right)=\varnothing$.

Définition 11 : On dit que $(\tilde{x}, \tilde{y}) \in X \times Y$ est un point de selle du triplet $[h, X, Y]$ si l'on a : $h(\tilde{x}, y) \leqq h(\tilde{x}, \tilde{y}) \leqq h(x, \tilde{y}) \forall(x, y) \in X \times Y$ c'est-à-dire : $\operatorname{Sup}_{y \in Y} h(\tilde{x}, y)=h(\tilde{x}, \tilde{y})=\operatorname{Inf}_{x \in X} h(x, \tilde{y})$.

THÉORÈme 8: Soient $k \in \overline{\mathbb{R}}^{X \times Y}$ de type $S, \varphi=P_{X} \theta k \in S\left(X \times Y^{\prime}\right)$ et $\psi=P_{X} k \in S\left(X^{\prime} \times Y\right)$. Le couple $(\tilde{x}, \tilde{y}) \in X \times Y$ est point de selle de $[k, X, Y]$ si et seulement si $\varphi\left(\tilde{x}, 0_{Y}\right)+\psi\left(0_{X}, \tilde{y}\right)=0$.

Preuve. - D'après la définition du point de selle, on $\mathrm{a}$ :

$$
f(\tilde{x})=\operatorname{Sup}_{y \in Y} k(\tilde{x}, y)=k(\tilde{x}, \tilde{y})=\operatorname{Inf}_{x \in X} k(x, \tilde{y})=g(\tilde{y}) .
$$

Or

$$
g(\tilde{y})=\theta P_{X} k\left(0_{X^{\prime}}, \tilde{y}\right)=-\psi\left(0_{X^{\prime}}, \tilde{y}\right)
$$

et

$$
f(\tilde{x})=P_{Y} \theta k\left(\tilde{x}, 0_{Y^{\prime}}\right)=\varphi\left(\tilde{x}, 0_{Y}\right),
$$

d'où le résultat suivant : $\varphi\left(\tilde{x}, 0_{Y^{\prime}}\right)+\psi\left(0_{X^{\prime}}, \tilde{y}\right)=0$. @

De nombreux auteurs se sont intéressés à la recherche de conditions suffisantes caractérisant les points de selle ( $c f .[3],[5],[6],[7], \ldots)$.

\section{SOUS-DIFFERENTIEL D'UNE FONCTION QCX-QCV}

Soit $k \in \overline{\mathbb{R}}^{X \times Y}$ de type $S$. Pour chaque $y$ fixé dans $Y$, la fonction : $x \mapsto k(x, y)$ est qcx. On notera $\partial_{X} k(x, y)$ son sous-différentiel en $x$. C'est une partie (éventuellement vide) de $X^{\prime}$. De même, pour chaque $x$ fixé dans $X$, la fonction : $y \mapsto-k(x, y)$ est qcx. On notera $\partial_{Y} k(x, y)$ son sous-différentiel en $y$. C'est une partie (éventuellement vide) de $Y^{\prime}$. 
Définition 12 : Soit $k \in \overline{\mathbb{R}}^{X \times Y}$ de type $S$.

L'ensemble $\partial k(x, y)=\partial_{X} k(x, y) \times \partial_{Y} k(x, y)$ est appelé le sous-différentiel de $k$ au point $(x, y)$. C'est une partie éventuellement vide de $X^{\prime} \times Y^{\prime}$.

THÉORÈME 9 : Soient $k \in \overline{\mathbb{R}}^{X \times Y}$ de type $S, \varphi=P_{Y} \theta k$ et $\psi=P_{X} k$. $\left(x^{\prime}, y^{\prime}\right) \in \partial k(x, y) \Leftrightarrow \varphi\left(x, y^{\prime}\right)+\psi\left(x^{\prime}, y\right)=0$ et $\left(x^{\prime}, y^{\prime}\right) \in P(x) \times P(y)$.

Preuve : Il suffit d'utiliser la définition (4). En effet :

$$
\begin{array}{ccccc}
x^{\prime} \in \partial_{X} k(x, y) \Leftrightarrow & x^{\prime} \in P(x) \quad \text { et } & P_{X} k\left(x^{\prime}, y\right)+k(x, y)=0 \\
y^{\prime} \in \partial_{Y} k(x, y) \Leftrightarrow & y^{\prime} \in P(y) \quad \text { et } & P_{Y} \theta k\left(x, y^{\prime}\right)+\theta k(x, y)=0 . \\
k \text { est de type } S & \Rightarrow & \varphi \in S\left(X \times Y^{\prime}\right) & \text { et } & \psi \in S\left(X^{\prime} \times Y\right) .
\end{array}
$$

Ainsi

$$
\left(x^{\prime}, y^{\prime}\right) \in \partial k(x, y) \Rightarrow\left(x^{\prime}, y^{\prime}\right) \in P(x) \times P(y)
$$

et

$$
P_{Y} \theta k\left(x, y^{\prime}\right)+P_{X} k\left(x^{\prime}, y\right)=0,
$$

c'est-à-dire

$$
\left(x^{\prime}, y^{\prime}\right) \in \partial k(x, y) \Rightarrow\left(x^{\prime}, y^{\prime}\right) \in P(x) \times P(y)
$$

et

$$
\varphi\left(x, y^{\prime}\right)+\psi\left(x^{\prime}, y\right)=0 .
$$

Inversement supposons que

$$
\varphi\left(x, y^{\prime}\right)+\psi\left(x^{\prime}, y\right)=0 \quad \text { et } \quad\left(x^{\prime}, y^{\prime}\right) \in P(x) \times P(y) .
$$

Par définition,

$$
-\psi\left(x^{\prime}, y\right)=\operatorname{Inf}\left\{k(u, y) / u \in P\left(x^{\prime}\right)\right\} .
$$

Comme $\quad x^{\prime} \in P(x) \Rightarrow x \in P\left(x^{\prime}\right)$ on déduit l'inégalité suivante : $\psi\left(x^{\prime}, y\right)+k(x, y) \geqq 0$.

De même,

$$
-\varphi\left(x, y^{\prime}\right)=\operatorname{Inf}\left\{-k(x, v) / v \in P\left(y^{\prime}\right)\right\} .
$$

Comme $y^{\prime} \in P(y) \Rightarrow y \in P\left(y^{\prime}\right)$, on déduit l'inégalité suivante :

$$
\varphi\left(x, y^{\prime}\right)+\theta k(x, y) \geqq 0 .
$$


Et d'après (5), on déduit que :

$$
\psi\left(x^{\prime}, y\right)+k(x, y)=0 \quad \text { et } \quad \varphi\left(x, y^{\prime}\right)+\theta k(x, y)=0 .
$$

THÉORÈME 10: Soient $k \in \overline{\mathbb{R}}^{X \times Y}$ de type $S, K$ la classe de $k$ et $m \in M=K^{*}$. Alors $\left(x^{\prime}, y^{\prime}\right) \in \partial k(x, y) \Leftrightarrow(x, y) \in \partial m\left(x^{\prime}, y^{\prime}\right)$.

\section{Preuve : Soit}

$$
\begin{aligned}
& m \in M \cdot \underline{m} \leqq m \leqq \bar{m} \Rightarrow \theta \bar{m} \leqq \theta m \leqq \theta \underline{m} \quad \Rightarrow \quad P_{Y^{\prime}}, \theta \underline{m} \leqq P_{Y^{\prime}}, \theta m \leqq P_{Y^{\prime}}, \theta \bar{m} \\
& \text { (resp. } P_{X}, \bar{m} \leqq P_{X^{\prime}} m \leqq P_{X}, \underline{m} \text { ). }
\end{aligned}
$$

Comme

$$
\begin{gathered}
P_{Y}, \theta \bar{m}=P_{Y}, \theta \theta P_{Y} \psi=P_{Y^{\prime}}, P_{Y} \psi=\psi \quad \text { et } \quad P_{Y^{\prime}}, \theta \underline{m}=P_{Y^{\prime}}, \theta P_{X} \varphi=\psi \\
\text { (resp. } \left.P_{X}, \bar{m}=P_{X^{\prime}}, \theta P_{Y} \psi=\varphi \text { et } P_{X}, \underline{m}=P_{X^{\prime}} P_{X} \varphi=\varphi\right),
\end{gathered}
$$

on déduit que $P_{Y^{\prime}}, \theta m=\psi \in S\left(X^{\prime} \times Y\right)$ (resp. $\left.P_{X^{\prime}} m=\varphi \in S\left(X \times Y^{\prime}\right)\right)$. Il suffit d'appliquer le théorème précédent et la définition (4) à $m$ :

$$
\begin{aligned}
& \left(x^{\prime}, y^{\prime}\right) \in \partial k(x, y) \\
& \quad \Leftrightarrow \varphi\left(x, y^{\prime}\right)+\psi\left(x^{\prime}, y\right)=0 \quad \text { et } \quad\left(x^{\prime}, y^{\prime}\right) \in P(x) \times P(y) . \\
& \Leftrightarrow P_{X^{\prime}} m\left(x, y^{\prime}\right)+P_{Y^{\prime}}, \theta m\left(x^{\prime}, y\right)=0 \quad \text { et } \quad(x, y) \in P\left(x^{\prime}\right) \times P\left(y^{\prime}\right) .
\end{aligned}
$$

Or

$$
\begin{aligned}
P_{X^{\prime}} m\left(x, y^{\prime}\right)+m\left(x^{\prime}, y^{\prime}\right)+\theta m\left(x^{\prime}, y^{\prime}\right)+P_{Y^{\prime}} \theta & m\left(x^{\prime}, y\right) \\
& =P_{X^{\prime}} m\left(x, y^{\prime}\right)+P_{Y^{\prime}} \theta m\left(x^{\prime}, y\right)=0
\end{aligned}
$$

et comme on a toujours

$$
P_{X^{\prime}} m\left(x, y^{\prime}\right)+m\left(x^{\prime}, y^{\prime}\right) \geqq 0 \quad \text { et } \quad \theta m\left(x^{\prime}, y^{\prime}\right)+P_{Y^{\prime}} \theta m\left(x^{\prime}, y\right) \geqq 0
$$

on déduit que

$$
P_{X^{\prime}} m\left(x, y^{\prime}\right)+m\left(x^{\prime}, y^{\prime}\right)=0 \quad \text { et } \quad \theta m\left(x^{\prime}, y^{\prime}\right)+P_{X^{\prime}}, \theta m\left(x^{\prime}, y\right)=0
$$

ce qui est équivalent à $(x, y) \in \partial m\left(x^{\prime}, y^{\prime}\right)$. @ 


\section{BIBLIOGRAPHIE}

1. M. Atreia et A. Elqortobi, Quasi-Convex Duality, Lectures Notes in Control and Inform. Sci. Optimization and Optimal Control, 1980, 30, p. 16-22.

2. A. Elqortobi, Thèse de $3^{\mathrm{e}}$ cycle, $\mathrm{N}^{\circ}$ d'ordre 2312, Université Paul-Sabatier, Toulouse, 1980.

3. P. J. Laurent, Fonctions convexes et problèmes de Minimax. Séminaire d'Analyse Numérique, Grenoble, 1972.

4. P. J. Laurent, Approximation et Optimization, Hermann, Paris.

5. L. Mclinden, A Minimax Theorem, Math. Op. Res., nov. 1984, 9, nº 4.

6. H. Nikaido, On Von Neumann's Minimax Theorem, Pacific J. Math., 1954, 4, p. 65-72.

7. U. PAssy et E. Z. Prisman, Saddle-functions and min-max problem. The QuasiConvexe Quasi-Concave Case, Mimeograph series $n^{\circ} 299$, Faculty of Industrial Engineering and Management, Technio, Haifa, Israel, 1981.

8. R. T. Rockafellar, Convex Analysis, Princeton, 1970.

9. M. Sıon, On General Minimax Theorems, Pacific J. Math., 1958, 8, p. 171-176.

10. M. Volle, Conjugaison par tranches, Ann. Mat. Pura Appl., 1985, IV, 139, p. 279 312. 